\title{
RECENT MEMORY AND PERFORMANCE IMPROVEMENTS IN OCTOPUS CODE
}

\author{
Joseba Alberdi-Rodriguez ${ }^{1,2,3}$, Micael J. T. Oliveira ${ }^{3,4}$, Pablo García-Risueño ${ }^{5}$, \\ Fernando Nogueira ${ }^{3}$, Javier Muguerza ${ }^{1}$, Agustin Arruabarrena $^{1}$, and Angel \\ Rubio $^{2,6,7}$ \\ 1 Dept. of Computer Architecture and Technology, University of the Basque Country \\ UPV/EHU, M. Lardizabal, 1, 20018 Donostia-San Sebastián, Spain \\ 2 Nano-Bio Spectroscopy Group and European Theoretical Spectroscopy Facility, \\ Spanish node, University of the Basque Country UPV/EHU, Edif. Joxe Mari Korta, \\ Av. Tolosa 72, 20018 Donostia-San Sebastián, Spain \\ 3 Center for Computational Physics, University of Coimbra, Rua Larga, 3004-516 \\ Coimbra, Portugal \\ 4 Unité Nanomat, Université de Liège, allée du 6 août, 17, B-4000 Liège, Belgium \\ 5 Institut für Physik und IRIS Adlershof, Humboldt Universität zu Berlin, Zum \\ Grossen Windkanal 6, 12489 Berlin, Germany \\ 6 Centro de Física de Materiales, University of the Basque Country UPV/EHU, \\ 20018 Donostia-San Sebastián, Spain \\ 7 Fritz-Haber Institut der Max-Planck Gesellschaft, Faradayweg 4-6, D-14195 \\ Berlin-Dahlem, Germany
}

\begin{abstract}
In this work we present the improvements made to the OCTOPUs code in order to reduce the memory requirements and to optimise parallel data distribution. Both topics are central for efficiency and feasibility of calculations when the system must be run in a large HPC environment. These modifications were mainly made in the real-space mesh partitioning and mapping algorithms, and are thus transferable to other codes using this type of real-space representation of data. The code became much more efficient, and we present several scalability results showing that it is now possible to address ab-initio quantum-mechanical simulations of the interaction of light with big biomolecules, paving the way for a better understanding of phenomena such as energy conversion in plants.
\end{abstract}

Keywords: DFT, TDDFT, OCTOPUs software, HPC, memory optimisations.

\section{INTRODUCTION}

The understanding of phenomena at nanoscopic scale is a major goal of science since the very moment of the appearance of quantum physics. Software simulation is an invaluable tool to study these types of phenomena. Currently, high performance computing (HPC)[1] enables the simulation of atomic and molecular systems according to the fundamental equations of quantum mechanics. 
OCторUs $[2-4]$ is a popular software for quantum electronic structure calculations. It is based on Density Functional Theory (DFT) $[5,6]$ and Timedependent Density Functional Theory (TDDFT) [7,8], which are the most widely used approaches in the field of quantum ab-initio simulation.

In this work we will present recent memory improvements in the OCTOPUS code and we will show its extreme performance. Previous to this work, OCTOPUs was prepared to run with relatively small atomic systems (hundreds of atoms at most), in machines with hundreds of processor cores. In the aim to reach bigger systems with thousands of atoms, it is necessary to use also thousands of processors, and this is the scenario where new problems appear. Altough the results to be presented are limited to OCTOPUs, lessons learned in this work can be readily applied to any other real-space mesh based code. It is to be stressed that our data transfer improvements correspond to the calculation of the Hartree potential, which is customarily calculated using real-space meshes also for codes using basis sets.

This paper is structured as follows. In section 2 we give some comments on how the OCTOPUs code works. In section 3, we explain the strategies we followed to increase the efficiency and optimise the memory management. Some measurements on the current performance of the code are presented in section 4. Finally, in section 5 we outline the main conclusions of our work.

\section{INTRODUCTION TO THE OCTOPUS CODE}

The simulation at a quantum level of systems consisting of thousands of atoms makes it possible to understand a wide variety of physical, chemical and biological phenomena. Despite the remarkable recent improvements in scientific codes and HPC infrastructures, the size of the systems that can be simulated using TDDFT is still very limited, and performing calculations with thousands of atoms is still a significant challenge.

Осторus is a very efficient scientific software package used to study by first principles the properties of the excited states of large biological molecules, complex nanostructures, and solids. The code is mostly developed for density functional theory (DFT) and time-dependent density functional theory (TDDFT) calculations, which are convenient quantum-mechanic approaches to study the electronic structure of molecular systems and its time evolution behaviour. It has been proven that their capacity to provide accurate results on the description of a big variety of phenomena at a relatively cheap computational cost; specially, TDDFT is being used to accurately predict, ab-initio, the optical absorption spectra of biological systems. When properly validated, TDDFT calculations can be quite reliable, and they are increasingly used by non-experts to support and interpret experimental results.

In TDDFT the main quantities to be represented are three-dimensional functions: the density and the single particle orbitals (Kohn-Sham states). For big systems these are the most memory demanding variables. Octopus uses MPI and OpenMP for parallelisation. For several tasks the code relies on external 
libraries. For example, linear algebra operations are handled using the BLAS and LAPACK libraries, and the Poisson equation is solved using very efficient massively parallel libraries (either interpolating scaling functions (ISF) [9] or parallel fast Fourier transforms (PFFT) [10]). Also the Laplacian of the states has to be evaluated for every mesh point. OCTOPUs calculates it by finite differences, usually using a star-stencil with 24 neighbours. Support for BLACS and SCALAPACK is also available, but it has not been optimised yet.

Oсторus is released under the GPL license, so it is freely available to the whole scientific community for use, study and modification. The code has been developed extensively in the last years to study systems up to hundreds of atoms, and it is a present goal to make it suitable for systems of thousands of atoms. Over the past years, OcTOPus has evolved into a fairly complex and complete tool, and it is now being used by dozens of research groups around the world.

\subsection{DATA STRUCTURES}

As mentioned above, in TDDFT the main quantities to be represented are threedimensional functions: the electronic density and the single particle orbitals (states). The single particle orbitals are evolved following the time-dependent Kohn-Sham equations [6] taking as initial condition in most cases the solution of the ground-state density functional theory problem, also obtained by OcTOPUs. In the code the functions are represented in a real-space mesh, and differential operators are approximated by high-order finite differences. OCTOPUs is able to combine multi-level parallelism by using MPI for coarse grain parallelism and OpenMP for a finer level. It can also take advantage of GPU architectures. The MPI parallelisation relies on a tree-based data parallelism approach. The main piece of data to be divided among processes are the single particle states, an object that depends on two main indices: the state index and the space coordinate. Each one of these indices is associated with a data parallelization level, where each process is assigned a section of the total range of the index. There is also a third index, which defines the k-point and spin values. This multi-level parallelization scheme ensures a very good scaling of real-time TDDFT.

As the size of the system grows, two factors affect the computing time: first, the space region to simulate is larger, and second, there are more electrons to simulate (which is directly related to the number of electronic states). By dividing each of these degrees of freedom among processors, multi-level parallelization ensures that the total parallel efficiency remains constant as we increase the system size and the number of processors.

MESH. The mesh is the data structure used to represent the space. The realspace has to be bounded and discretised, and it can be done in one, two or three dimensions. On top of this mesh are represented such things as the potential energy $(\nu)$, the electronic density of the system $(\rho)$, the system wavefunction, etc.

The shape of the mesh data structure can be adaptive, parallelepiped or spherical (Figure 1). In the former case (which is the default) the mesh is made 
by an union of spheres centred in the atoms of the simulated system; in the other two cases the shape is regular: a parallelepiped and a sphere, respectively. Two parameters define the mesh: the radius (from each atom in the adaptive case; the length of each edge in the parallelepiped case; and from the centre in the case of the sphere) and the spacing, which is the distance between two consecutive mesh points. All the mesh points are usually taken to be equally spaced.

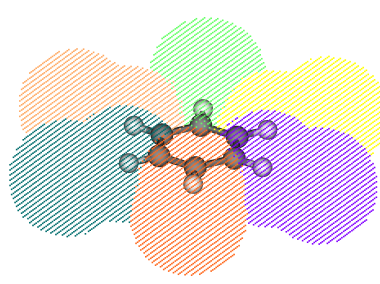

Adaptive

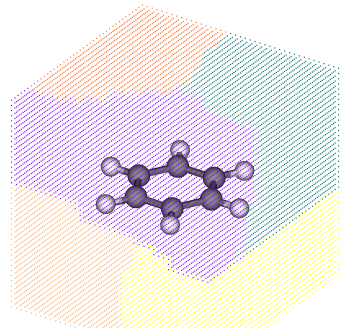

Parallelepiped

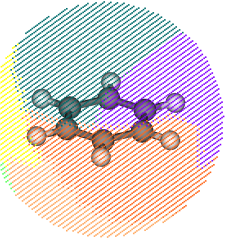

Spherical

Fig. 1. Different mesh partitions for the benzene molecule. The mesh is partitioned in 6 domains using adaptive, parallelepiped and spherical shapes.

STATE. A state is the representation of an electronic orbital. Each one of the electronic states under study is defined by a concrete data structure, which is represented over the mesh.

A lower bound of the memory requirements of the simulation can be estimated from the number of simulated states $\times$ number of mesh points.

CUBE. The cube is an auxiliary data structure used to solve Fourier transforms (FFTs), which are needed to calculate the electrostatic Hartree potential. The evaluation of FFTs in 3D demands to represent the data in parallelepiped meshes. In addition, the cutoff technique [11] used in OCTOpus to reduce the effect of the undesired periodic images of the inverse FFT also requires cubic meshes.

The edge of the cube is by default twice the length of the largest axis of the mesh; thus, it is at least 8 times bigger than the corresponding mesh. The cube is filled with the mesh points (explained in the next section), and all the extra points are padded with zeros.

\subsection{EXECUTION MODES}

OCTOPUs simulates physical systems basically in two phases: firstly, the electronic ground-state is calculated, and secondly, the converged Kohn-Sham wavefunctions are propagated in time under the effect of an external perturbation. 
The ground-state (GS) is obtained applying density functional theory (DFT), whereas the TDDFT theory is used to obtain the time-dependent (TD) solution.

Memory needs increase (roughly) quadratically with the system size under simulation. Generally, the GS requires the use of real numbers for quantum states, while the TD runs require the use of complex numbers. Thus, the amount of memory in a time-dependent run usually doubles that of the corresponding ground-state. On the other hand, total computation amount (FLOP) does not increase in the same way for the two modes: the ground-state increases roughly as $S^{5 / 2}$, while the time-dependent increases as $S^{3 / 2}$ ( $\mathrm{S}$ is the system size, i.e., the number of atoms).

These two run modes scale differently with respect to the number of processes, and pose different problems. More precisely, the time-dependent run mode scales better than the ground-state calculation, as we will see in the next section. The different scalabilities are due to the specific parallelization schemes available in each run mode.

\section{IMPROVEMENTS IN THE MEMORY USAGE}

Octopus has been used successfully during the last years to analyse complex nanostructures, but it had some limitations when a high number of processes must be used to simulate systems with thousand of atoms. Most of the limitations are related to the memory requirements, which is a limited resource in any computer. So, we have analysed how OCTOPUs uses the computer memory, to optimise its usage.

\subsection{MESH PARTITIONING}

The largest data structures, namely the functions represented on the mesh, have to be distributed between the available computer nodes. An important issue in this domain parallelisation is selecting which points of the mesh are assigned to each processor. This task, known as mesh partitioning, is not trivial for meshes of adaptive shape. Not only the number of points must be balanced between processors but also the number of points in the boundary regions must be minimised. This issue is crucial, because communication costs are directly related to the number of boundary points. An example of a mesh partitioning is shown in Figure 1 (each colour represents a domain). OCTOPUs relies on external libraries for this task: Metis [12] and ParMetis [13]. These libraries implement several algorithms and the quality of the partition will depend on the selected one.

SERIAL PARTITIONING. Previous to this work, Metis was the default library in OCTOPUs to partition the mesh. It is a serial library, so the process that calls it does need all the mesh data, which have to be gathered from all the

processes. Moreover, to reduce communication needs, all the processes called the library and, consequently, all of them needed to store the whole data structure. 
This is a valid approach if small-medium size meshes are used, but it is unfeasible with million of mesh points, because the amount of memory per process is limited.

PARALLEL PARTITIONING. With the aim of avoiding the mentioned memory problems with the mesh partitioning, we have adapted OCTOPUs to use a new a highly parallel library called PARMetis. PARMetis is a parallel library implemented in MPI and built on top of the MeTis library (version 5.1). As METIs, this library makes partitions of graphs, so a transformation of the OCTOPUs mesh structure into a graph is required previously. We use a graph data structure to store the neighbour information of all points. Therefore, each mesh point is represented by a vertex in the graph, and neighbours points are connected through an edge. The information of which the neighbours of a point are is necessary e.g. to calculate the discretised Laplacian operator, which is done using a stencil (a given function of the values of a function in a given point and in its neighbours). PARMETIS works in parallel and, so, all data structures are distributed. Each process works with a contiguous chunk of the graph, with local matrices of vertexes and edges, and all the processes know how the graph is initially distributed.

In order to use the new library, we have developed a new distributed version of the mesh partitioning for OCTOPUs. At the beginning, each process obtains an arbitrary (not optimised) portion of the mesh (called "division"), whose size is roughly $N / P$ (being $N$ the number of mesh points and $P$ the number of MPI processes involved in the partitioning). Then, using this initial division, each process calls PARMETIS to obtain the actual domain partition of the graph. Finally, local results are informed to the corresponding processes (the real owners of the mesh points).

So, the final domain partition of the mesh is saved in a distributed way. Consequently, when a process works with a neighbour point that does not belong to its partition, it must identify the owner of the point. This information can be obtained from the initial owner of the points, that processed it using PARMETIS.

Figure 2 shows an example of this procedure. Let us assume that we are working with the blue mesh point with the circle inside, point [3,4], belonging to domain 2 (process 2 ) and labelled as working point. Using a stencil of length 2 , it wants to obtain the two neighbours on the right (points $[4,4]$ and $[5,4]$ ). According to the initial division of the mesh, it knows that process 1 (division 1) calculated the final owner of those points. So, it will ask process 1 for this information (both points are assigned to process 3 ) before doing the actual communication.

This new distribution strategy, based in PARMetis, allows us to use only local data to do the final partition/decomposition, reducing greatly the use of memory in each process. 


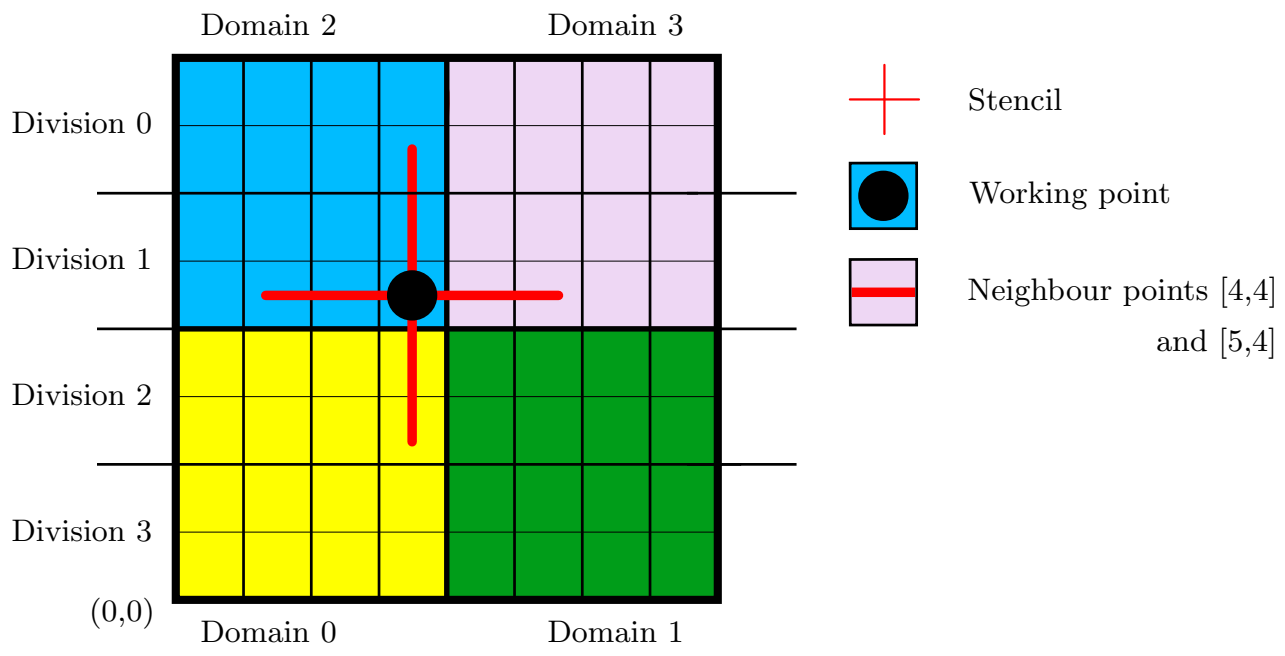

Fig. 2. A simplified example of the Octopus mesh partition. The initial (nonoptimised) division schedules chunks of $2 \times 8$ points (division $i$ is assigned to process $i$ ). Afterwards, each process uses PARMETIS locally to create the actual domain partitions ( $4 \times 4$ points), aiming to minimize the boundary points.

\subsection{DATA TRANSFERS BETWEEN DIFFERENT PARTITION TYPES}

In the context of scientific simulation it is common to use external libraries to do standard operations in an highly optimised way. These libraries could use different data distributions. One example of this situation are the different mesh partitions used in OCTOPus and in its Poisson solvers [14] (the interpolating scaling functions (ISF) [9] and the parallel fast Fourier transforms (PFFT) [10] libraries).

Both Poisson solvers are based on FFT methods, where the spatial functions are represented in a cubic mesh. The ISF library splits the mesh into domains which are parallel plane-like parallelepipeds (Figure 3C), while PFFT makes a two-dimensional split of the mesh into column-like domains (Figure 3B). However, Осторus divides the mesh in a three-dimensional manner into more compact domains (Figure 3A). Therefore, the data that a process deals with in the OсторUs main program are not the same as the PFFT and ISF libraries use inside, and hence a data transfer is necessary, independently of the shape of the mesh.

The simple way to carry this data transfer out is to gather all the data (density $\rho$ or potential $v$ ) in all the processes before distributing them accordingly to the new mesh partition. In fact, this was the option used in the previous version of ОсторUs. This solution works if the number of processes is not very high, but efficiency decays when the number of processes increases, because of 
the global MPI communication. Therefore, a new data transfer strategy had to be implemented to be used in massively parallel machines.

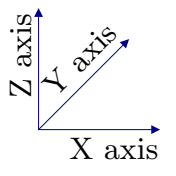

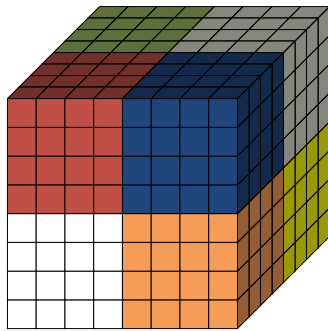

A) Octopus mesh

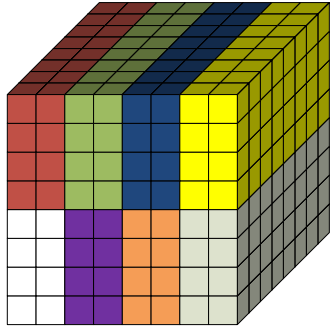

B) PFFT mesh

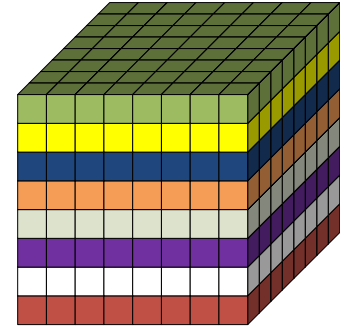

C) ISF mesh

Fig. 3. Simplified domain decomposition of the simulation mesh. Each little cube represents a mesh point $\left(8^{3}\right.$ points in total) and each colour represents a partition (8 domains). A) OCTOPUs mesh with a 3D domain decomposition; B) PFFT mesh with a 2D decomposition; C) ISF mesh with a 1D decomposition.

We have overcome this problem in a highly efficient way. At the initialisation stage a mapping between the OCTOPUs mesh partition and the FFT mesh partition is established and saved. This mapping is used when running the actual solver to efficiently communicate only the strictly necessary data between processes. Each of the different domain decomposition (for instance, Octopus and FFT partitions) is represented by a MPI group/communicator, that might differ in the number of processes (OCTOPUs might use processes for different parallelisation levels), but includes all the mesh points. At a given time, data points have to be sent from one group to the other. Unfortunately, MPI does not allow to send information between different groups unless they are disjoint, which is not, by definition, the current case. This means that communication will have to be done inside one of the groups. This is not a problem, because we can determine the rank of the receiver processes through the MPI_COMM_WORLD global communicator. Therefore, all the data transfers between processes can be done in a unique MPI_Alltoallv function. It is important to note that, using this improvement, each process only transfers each point once. Therefore, the total amount of information that must be sent between all the processes is equal to the number of points in the mesh, and it is independent of the number of processes. The data transfer process has been encapsulated in an specific Fortran module in Octopus, ${ }^{\mathrm{h}}$ achieving almost perfectly linear parallel scaling.

\footnotetext{
${ }^{\mathrm{h}}$ See the source code of our implementation in the file src/grid/mesh_cube_parallel_map.F90 at public Subversion repository: http://www.tddft.org/svn/octopus and the documentation in the wiki: http://www.tddft.org/programs/octopus).
} 


\subsection{OTHER IMPROVEMENTS}

Apart from the memory optimisations explained above, the older version of the code used as temporary arrays three matrices of size $P \times P$, being $P$ the number of running processes. Those matrices are small with few processes, but they become problematic when we need to use a high number of processes $>2048$. Assuming 8 byte integers, with 2048 processes the size of each matrix is of 32 $\mathrm{MiB}$, yet not very large, but with 8192 processes the size increases to $0.5 \mathrm{GiB}$, and with $64 \mathrm{~K}$ processors the size is already prohibitive $(32 \mathrm{GiB})$ for current machines. After implementing a new logic, we got rid of them, because they were only used in the initialisation process.

Other inefficiencies related to initialisation processes (serial evaluation of the local and boundary points, for example) also had impact in the execution time when simulating big systems, and have been removed.

\section{RESULTS}

Together with the optimization of the data flow, the main goal of this work was to address the huge memory requirements of ОСторUs when running in a large HPC environment. The changes made in the code allow it to run in a much more efficient manner, opening the door for the simulation of the interaction of light with big biomolecules. In this section we will first present the improvements in the memory usage, and next will proceed to demonstrate the extreme scalability of the code in its current form. No scalability tests for older versions of the code will be shown, as they were not able to handle the largest systems discussed below.

Tests have been done for different chunks of the light harvesting complex molecule, of 180, 650, 1365 and 2676 atoms (Figure 4). Those systems have from 452,878 mesh points and 250 electronic states to $4,106,680$ points and 3,656 states.

Tests were run using three different computers: a Blue Gene/P, a Blue Gene/Q and a small cluster (Corvo).

- The Blue Gene/P (BG/P) [15] system uses low power processors (IBM PowerPC 450, running at $850 \mathrm{MHz}$ ) connected with highly efficient communication networks. Each node has one chip of 4 cores, with only 2 GiB of RAM. In its largest installation it had 294,912 processor cores.

- The Blue Gene/Q (BG/Q) [16] is the next generation of the BG systems. Each compute card (which we call a compute node) of a BG/Q features an IBM PowerA2 chip with 16 cores working at a frequency of $1.6 \mathrm{GHz}, 16$ $\mathrm{GiB}$ of RAM and the network connections. A total of 32 compute nodes are plugged into a so-called node card. Then 16 node cards are assembled in one midplane, which is combined with another midplane and two I/O drawers to give a rack with a total of $32 \times 32 \times 16=16,386$ cores for each rack.

- The cluster Corvo represents a common parallel machine of a research group. Each node of Corvo has two Intel Xeon with 6 cores each and 48 GiB of RAM. There are 960 cores in total, connected with an Infiniband network. 


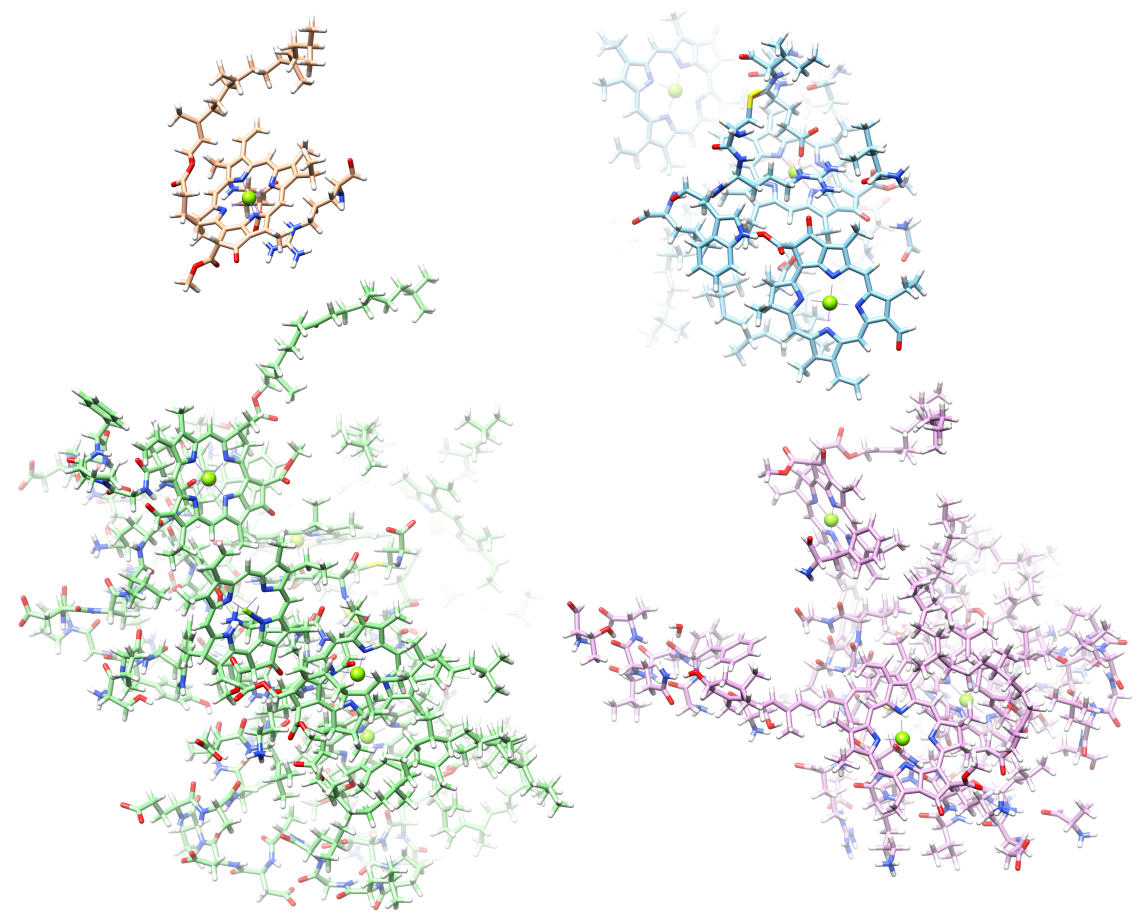

Fig. 4. Different chunks of the chlorophyll molecule, from 180 atoms to 2676 atoms.

We want to remark that the amount of memory per processor core is relatively low and it is not increasing significantly. For instance, in the computers that we have used, the quantity of memory per core goes from $0.5 \mathrm{GiB}$ in the $\mathrm{BG} / \mathrm{P}$ to $4 \mathrm{GiB}$ in Corvo, if all the cores are used to calculate.

\subsection{MEMORY MEASUREMENT}

OCTOPus provides a tool to measure the memory usage and the timing of the most important functions. A deep profiling of the memory usage has been done mainly using this internal profiler and validated with VALGRIND's MASSIF tool[17]. For example, when running the system of 180 atoms in 32 MPI processes, MASsIF estimates memory usage up to $96 \mathrm{MiB}$, whereas OCTOPUS estimates $88.5 \mathrm{MiB}$. This difference is owing to the fact that Octopus does not take into account the memory of linked libraries such as MPI, ISF, etc. Despite this small difference, we have used the internal profiler because its execution overhead is much lower than that of MAssif tool.

Figure 5 shows the memory usage per process of the system of 180 atoms, before and after the optimisations we have done. Memory usage per process is 


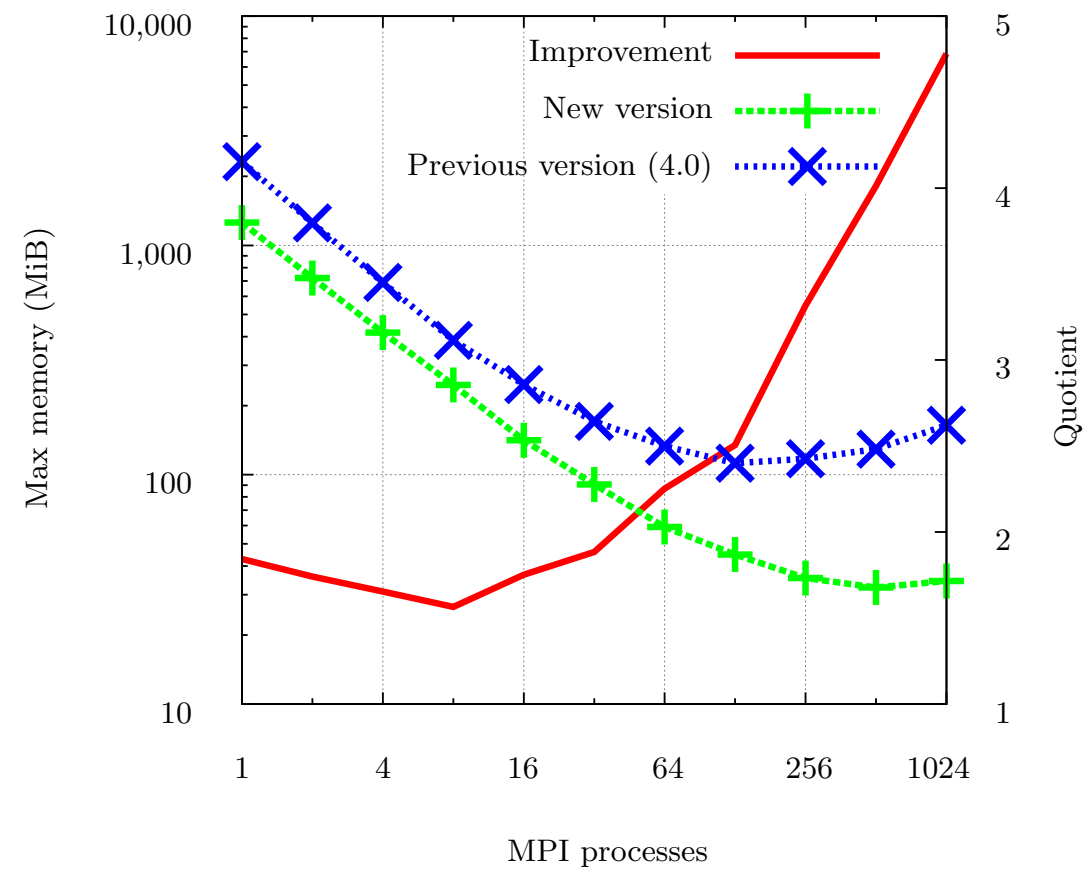

Fig. 5. Ground-state maximum memory usage (from OCTOPUs profiler) per MPI process for the system of 180 atoms.

decreasing as more processes are used, but unfortunately the oldest version (4.0) of OCTOPUs tends to increase the use of memory after a relatively small number of processes (256) and, so, makes impossible the use of thousands of processes. On the contrary, the last version has eliminated this increasing tendency. In fact, the memory need has been decreased by a factor of four in the studied range, with a clear tendency to be more efficient when using more processes. To realise the importance of this restriction it has to be considered that the amount of memory per CPU core hardly goes beyond $4 \mathrm{GiB}$.

\subsection{DATA TRANSFER TIME MEASUREMENT}

The new implementation of the mesh data transfer between different partitions (explained in section 3.2) shows really good performance as can be observed in the Figure 6 . The time spent transferring data is really low, compared with the execution time of the library (PFFT in this case). It is shown a particular case of a cubic mesh of edge 31.6 and spacing of 0.2 , which makes a total amount of 4,019,679 mesh points and needs $325^{3}=34,328,125$ cube points for the PFFT. The data transfer accounts from $0.5 \%$ to $55 \%$ of the total Poisson execution time. 


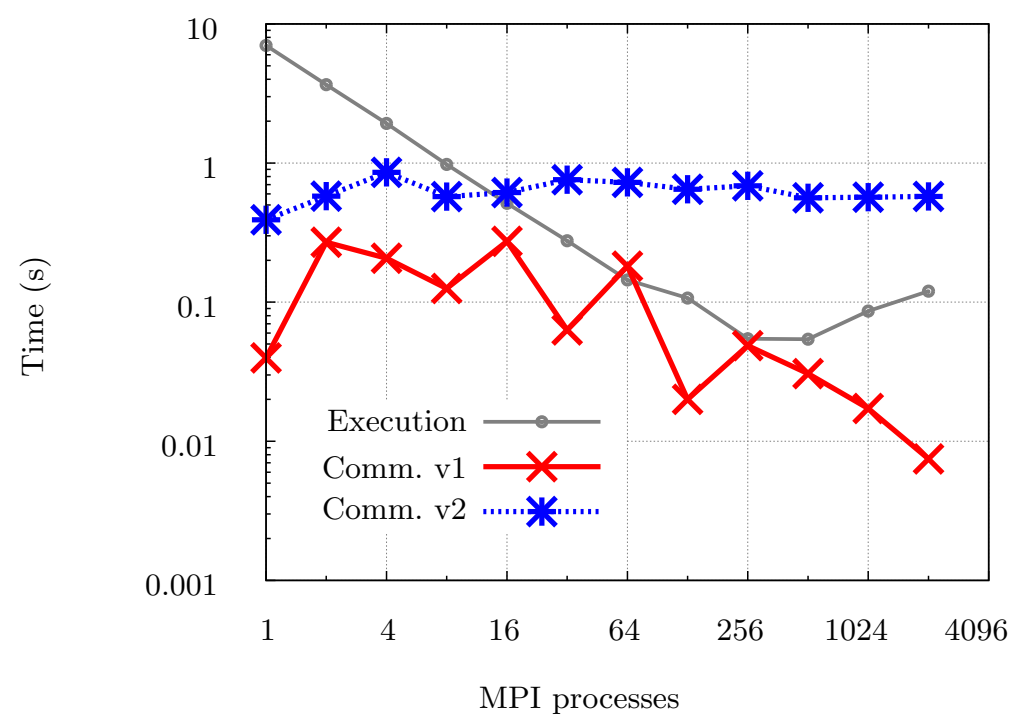

Fig. 6. Time for the new parallel communication pattern (Comm. v1), compared with the old serial communication (Comm. v2), for a system of 4,019,679 mesh points in the Blue Gene/P machine. While the old version does not scale and becomes a bottleneck, the improvement of the new approach is huge and it has a much better scalability. Total execution time of the Poisson solver is the sum of the Execution and Comm. times.

\subsection{EXECUTION TIMES}

Improvements made in the OCTOPUs code allowed us to simulate bigger systems than ever. Next paragraphs show the results obtained with both executions modes of OCTOPUs: ground-state and time-dependent.

GROUND-STATE. Figure 7 shows the execution time of the ground-state calculation of systems of 180, 441 and 650 atoms in Blue Gene/P and Corvo.

The trend for all the atomic systems and for both machines is equivalent, being Corvo 3 times faster. The scalability is more than acceptable; for instance, the 180 atoms system scales well up to 256 processes, while with the 650 atoms system the scalability is almost perfect until 256 processes, showing improvements up to 2048 MPI processes.

TIME-DEPENDENT. The execution times and relative speed-ups of the time-dependent simulations are show in the Figure 8. Our improvements in the OcTOpus code have allowed us to go further in the scalability and also to be able to run bigger systems. Executions correspond to the time-dependent in the BG/Q machine and to systems of 180, 650, 1365 and 2676 atoms. Runs are made using one MPI process per core, thus 16 processes per node. Extremely 


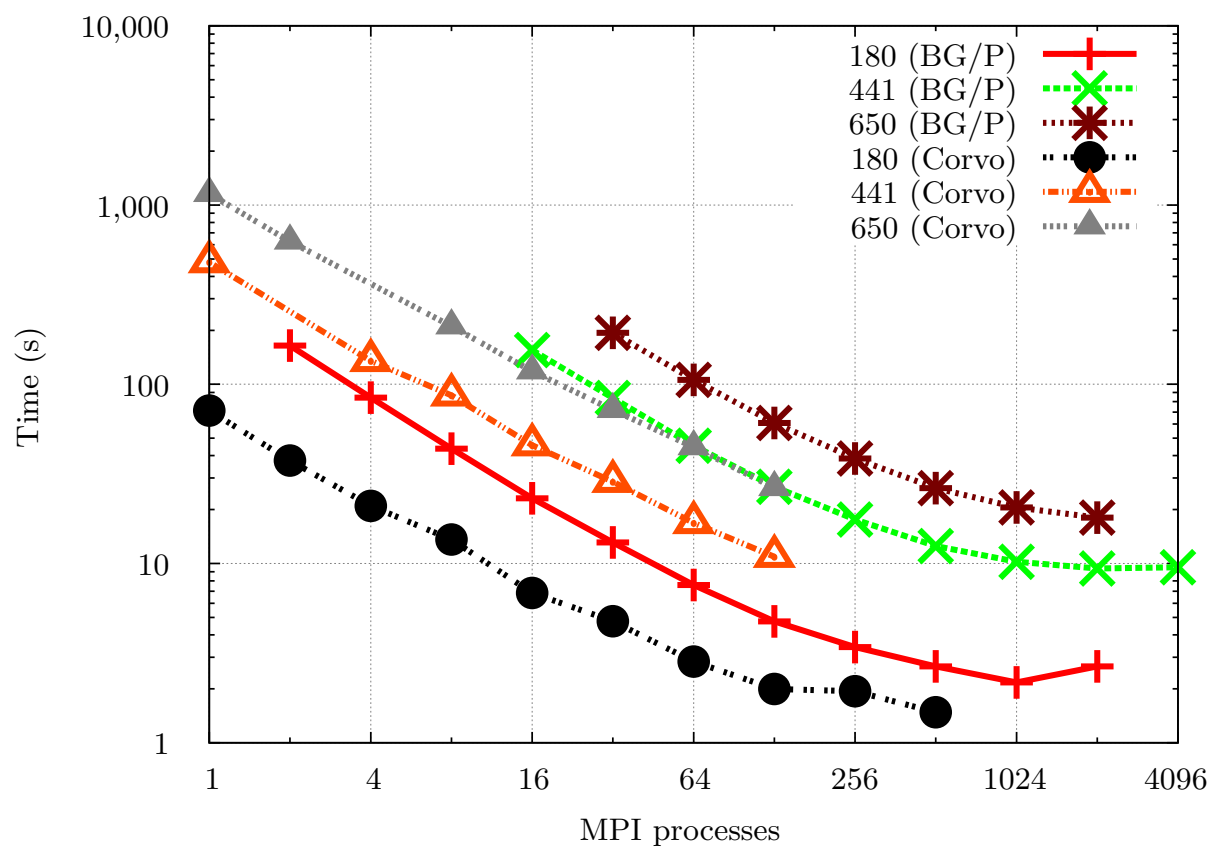

Fig. 7. Ground-state iteration execution time in Blue Gene/P and Corvo (x86-64)

good scalability is shown for the smallest system, that, because of the reduction of the memory needs, can be now run in only 4 MPI processes and it is still efficient with $8 \mathrm{k}$ processes. For the system of 650 atoms, there is no sign of saturation up to $16 \mathrm{k}$ processes. The system of 1365 atoms is highly parallel up to $32 \mathrm{k}$ processors and we have been able to run it in $64 \mathrm{k}$ processors, improving by far previous results. Finally, almost perfect scalability has been reached with the system of the 2676 atoms up to $16 \mathrm{k}$ processors (we were limited to the tests shown here, because we had a limited CPU quota for our project)..

Scaling tests also were done in the BG/P machine, showing a very similar behaviour. In this case, runs were made using one MPI process per node and four OpenMP threads (one per node core). Specially remarkable is the performance we have obtained with the 650 atoms system in this machine, which does not saturate until the maximum available CPU cores (128k). Deserves to mention as well the system of 180 atoms, which is really efficient from 4 up to $64 \mathrm{k}$ MPI processes. The new efficient memory usage allowed us to run in this machine a bigger system of 5879 atoms in $32 \mathrm{k}$ processors.

\section{CONCLUSIONS}

OcTOPus is a scientific software package based on TDDFT theory, which is being used successfully by dozens of research groups around the world. To date, it 

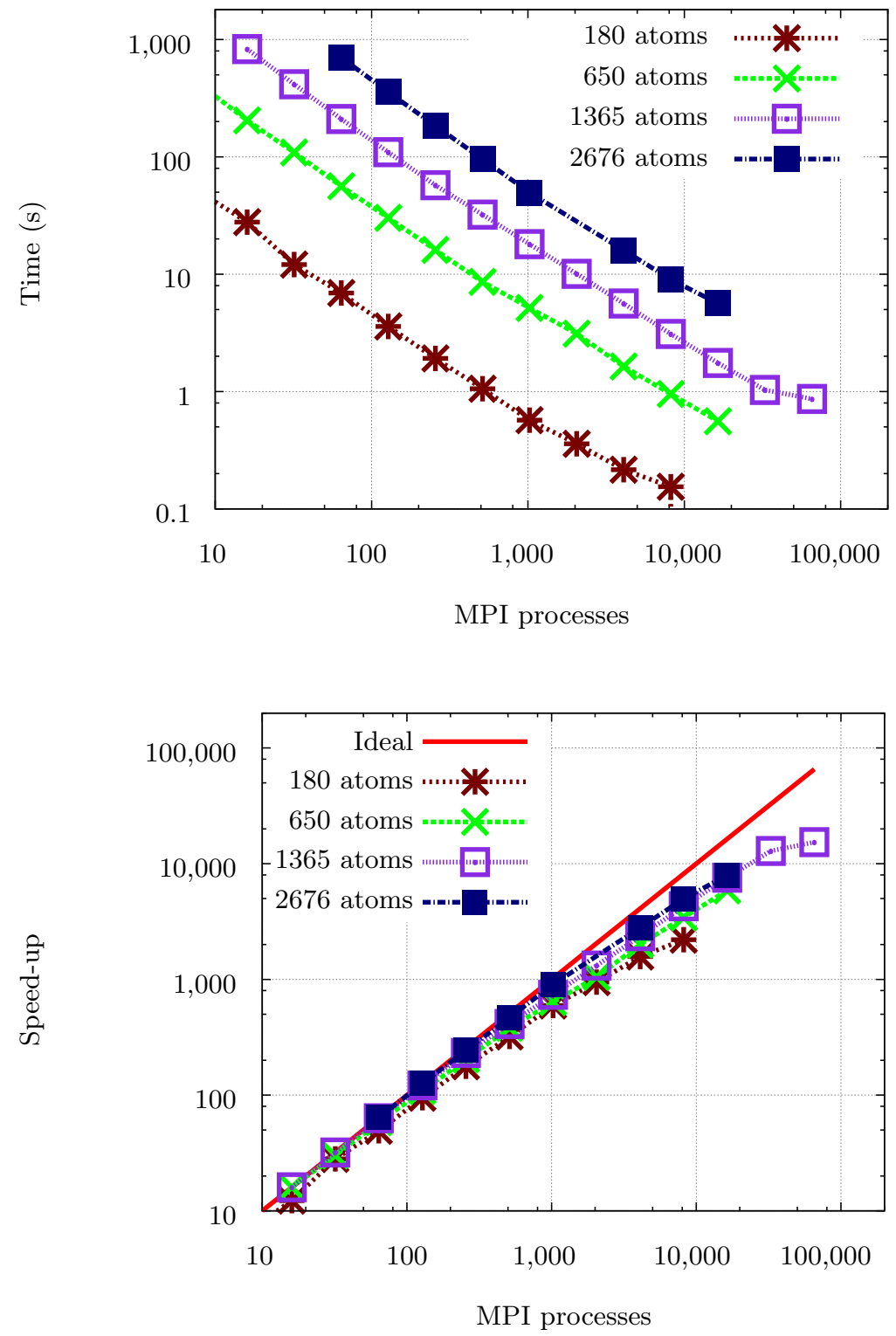

Fig. 8. 180, 650, 1365 and 2676 atoms system running in the Blue Gene/Q up to 65,536 MPI processes (1 MPI process per core).

was mostly used to analyse medium-sized complex nanostructures. Modifications were necessary to habilitate the code to work with bigger systems (thousands of atoms and beyond). 
In this work we have analysed and solved some of the limitations that OCTOPUs had in this new scenario, mainly the amount of memory and the transfers of the main data structures in some phases of the computation. Both problems have been solved in a very efficient manner. On the one hand, we have used PARMETIS to do the partitioning of the mesh using only local data, avoiding the use of the whole data mesh in each process and, consequently, reducing greatly memory needs. On the other, we have optimized data transfer between processors when different domain decompositions must be used.

The benefits of these improvements are significant. Осторus uses now a much more limited amount of memory per processor, and it is ready to simulate larger systems. Indeed, we have shown excellent scalability up to $64 \mathrm{k}$ and $128 \mathrm{k}$ cores, almost independent of the system size (number of atoms) beyond a given size. Although we have shown the executions of different chunks of the light harvesting complex, the real aim is to be able to run the entire light harvesting complex with more than 16,000 atoms, a work which is in progress. The recent improvements make possible to obtain scientifically relevant results for these large systems at a reasonable time.

Acknowledgements. This work was funded by the University of the Basque Country UPV/EHU (Aldapa, GIU10/02) and by the Department of Education, Universities and Research of the Basque Government (IT395-10 research group grant). We acknowledge financial support from the European Research Council Advanced Grant DYNamo (ERC-2010- AdG-267374), Spanish Grant (FIS201021282-C02-01), Grupos Consolidados UPV/EHU del Gobierno Vasco (IT578-13), Ikerbasque and the European Commission projects CRONOS (Grant number 280879-2). We acknowledge PRACE for awarding us access to resources Jugene and Juqueen based in Germany at JSC. J. Alberdi-Rodriguez acknowledges the scholarship of the University of the Basque Country UPV/EHU and the the support of project PRACE-3IP. P. García-Risueño is funded by the Humboldt Universität zu Berlin. M. J. T Oliveira acknowledges financial support from Belgian FNRS-FRFC project "Control of Attosecond Dynamics" number 2.4545.12.

\section{References}

1. García-Risueño, P., Ibáñez, P.E.: A review of high performance computing foundations for scientists. International Journal of Modern Physics C (IJMPC) (2012) 1230001

2. Andrade, X., Alberdi-Rodriguez, J., Strubbe, D., Oliveira, M., Nogueira, F., Castro, A., Muguerza, J., Arruabarrena, A., Louie, S., Aspuru-Guzik, A., et al.: Timedependent density-functional theory in massively parallel computer architectures: the octopus project. Journal of Physics: Condensed Matter 24(23) (2012) 233202

3. Castro, A., Appel, H., Oliveira, M., Rozzi, C.A., Andrade, X., Lorenzen, F., Marques, M.A.L., Gross, E.K.U., Rubio, A.: Octopus: a tool for the application of 
time-dependent density functional theory. Phys. Status Solidi (b) 243(11) (2006) $2465-2488$

4. Marques, M.A.L., Castro, A., Bertsch, G.F., Rubio, A.: Octopus: a first-principles tool for excited electron-ion dynamics. Comput. Phys. Commun. 151(1) (2003) 60-78

5. Hohenberg, P., Kohn, W.: Inhomogeneous Electron Gas. Phys. Rev. 136(3B) (1964) B864-B871

6. Kohn, W., Sham, L.J.: Self-Consistent Equations Including Exchange and Correlation Effects. Phys. Rev. 140(4A) (1965) A1133-A1138

7. Fiolhais, C., Nogueira, F., Marques, M.A.L., eds.: A Primer in Density Functional Theory. 1st edn. Springer, Series: Lecture Notes in Physics, Vol. 620 (2003)

8. Marques, M.A., Maitra, N.T., Nogueira, F.M.: Fundamentals of Time-Dependent Density Functional Theory. Volume 837. Springer Berlin Heidelberg (2012)

9. Genovese, L., Deutsch, T., Neelov, A., Goedecker, S., Beylkin, G.: Efficient solution of Poisson's equation with free boundary conditions. J. Chem. Phys. 125(7) (2006) 074105 Genovese, L. and Deutsch, T. and Neelov, A. and Goedecker, S. and Beylkin, G.

10. Pippig, M.: PFFT - An extension of FFTW to massively parallel architectures. SIAM J. Sci. Comput. 35 (2013) C213 - C236

11. Rozzi, C.A., Varsano, D., Marini, A., Gross, E.K.U., Rubio, A.: Exact coulomb cutoff technique for supercell calculations. Phys. Rev. B 73 (2006) 205119

12. Karypis, G., Kumar, V.: METIS - Unstructured Graph Partitioning and Sparse Matrix Ordering System, Version 2.0. Technical report (1995)

13. Karypis, G., Kumar, V.: Parallel multilevel k-way partitioning scheme for irregular graphs. In: Proceedings of the 1996 ACM/IEEE conference on Supercomputing (CDROM). Supercomputing '96, Washington, DC, USA, IEEE Computer Society (1996)

14. García-Risueño, P., Alberdi-Rodríguez, J., Oliveira, M.J.T., Andrade, X., Pippig, M., Muguerza, J., Arruabarrena, A., Rubio, A.: A survey of the performance of classical potential solvers for charge distributions. Journal of Computational Chemistry 35(6) (2014) 427-444

15. Sosa, C., Knudson, B.: IBM system Blue Gene solution: Blue Gene/P application development. IBM International Technical Support Organization (2008)

16. Gilge, M., et al.: IBM System Blue Gene Solution Blue Gene/Q Application Development. IBM Redbooks (2013)

17. Nethercote, N., Walsh, R., Fitzhardinge, J.: Building workload characterization tools with valgrind. In: Workload Characterization, 2006 IEEE International Symposium on, IEEE (2006) 2-2 